\title{
$\mathrm{OV} / 2-5$
}

\section{Theory and Observations of magnetic islands}

\author{
F. L. Waelbroeck \\ Institute for Fusion Studies, The University of Texas, Austin, Texas 78712, USA
}

\begin{abstract}
Islands are a ubiquitous feature of magnetically confined plasmas. They arise as the result of plasma instabilities as well as imperfections in the coils. Effective techniques have been developed for keeping their width small. Even thin islands, however, are observed to have nonlocal effects on the profiles of rotation and current. This has stimulated interest in using magnetic islands to control plasma transport. They are also of interest as a tool to improve our understanding of microscopic plasma dynamics.
\end{abstract}

\section{Introduction}

A goal shared by most magnetic confinement concepts is to realize a configuration consisting of simply-nested magnetic surfaces encircling a closed field line, the magnetic axis. In practice, small departures from this ideal configuration give rise to magnetic islands, which are tubes of flux with their own private magnetic axes. Their primary significance is that heat can flow rapidly around them by following the field lines. In the core, magnetic islands represent a loss of confinement volume. In the edge of some devices, however, magnetic islands serve a useful function by diverting the field so as to separate the hot plasma in the confinement volume from the material surfaces of the device.

The last few years have seen the successful development of RF current-drive techniques for reducing the size of magnetic islands. $[1,2,3,4,5,6,7]$ as a result, islands are no longer perceived as a direct threat to confinement, except in the context of ideal instabilities. Evidence has accumulated, however, showing that they have significant nonlocal effects on the profiles even when they occupy a small fraction of the confinement volume. [8, 9, 10, 11] This has fostered interest in using islands as agents to control transport and stability properties. In addition, islands constitute a useful diagnostic tool for obtaining information on the current and rotation profiles. Lastly, they provide a unique window into plasma dynamics at scales that are difficult to observe directly. Here, we present an overview of the experimental and theoretical results concerning magnetic islands.

\section{Magnetic islands as equilibrium structures}

We restrict attention to islands that are thin but sufficiently large to affect the background profiles of the reference equilibrium: in practice this corresponds to perturbation amplitudes in the range $10^{-4} \lesssim \delta B / B \lesssim 10^{-3}$. [12] The most direct approach to predicting the evolution of such islands is by using a general-purpose initial-value fluid 


\section{$\mathrm{OV} / 2-5$}

code. $[13,14,15,16,17]$ Due to the high conductivity of fusion plasmas, however, island widths generally evolve very slowly compared to the Alfvén time. Long simulation times are required for this reason as well as to address the question of the role of changes in the profiles. This, together with the spatial resolution requirements, has severely restricted the scope of the problems that can be treated in this way. A more adapted approach is to use the time and space-scale separation to advantage by investigating islands from the point of view of the equilibrium and transport of 3D helical plasmas. We elaborate on this approach.

In the context of equilibrium theory, the occurrence of magnetic islands is a consequence of the current conservation law, $\nabla \cdot \mathbf{J}=0$. Separating the components of the current in the directions parallel and perpendicular to the magnetic field, we note that the perpendicular component of the current, $\mathbf{J}_{\perp}$, is determined by force balance,

$$
\mathbf{J}_{\perp}=B^{-1} \hat{\mathbf{b}} \times \nabla p .
$$

Substituting the perpendicular current into Ampère's law, we find a magnetic differential equation for the parallel component of the current, $J_{\|}$:

$$
\mathbf{B} \cdot \nabla\left(J_{\|} / B\right)=-\nabla \cdot \mathbf{J}_{\perp} .
$$

It is convenient to use field-line coordinates defined by $\mathbf{B}=\nabla \chi \times \nabla(\zeta-q \theta)$, where $\theta$ and $\zeta$ are poloidal and toroidal coordinates, respectively, $\chi$ is a poloidal flux function, and $q$ is the safety factor. Fourier expansion using these coordinates yields the solution

$$
\left[\frac{J_{\|}}{B}\right]_{m, n}=\frac{\mu_{0} p^{\prime}}{\left\langle B^{2}\right\rangle} \sum_{m, n} \frac{\mathcal{G}_{m, n}(q)}{m-n q}+\hat{J}_{m, n} \delta(q-m / n),
$$

where $\mathcal{G}$ is a geometrical coefficient, the $m, n$ subscripts denote the Fourier coefficients of the corresponding variables, and the $\hat{J}_{m, n}$ are integration constants. Except in perfectly symmetric systems, the $\mathcal{G}_{m, n}$ do not vanish and the parallel component of the current is singular on every flux surface with a rational safety factor $q$.

As a consequence of plasma resistivity, the current singularities bring about magnetic reconnection and the breakup of the resonant magnetic surfaces into island chains. The primary object of island theory is to calculate the width and either the phase (for externally produced asymmetries) or the rotation frequency (for spontaneous symmetry-breaking bifurcations) of the resulting islands. It is important to keep in mind, however, that the width of the island is not merely a local property but that it measures the amplitude of the "tearing" component of the wavefunction in the entire device. In fact, islands in the core were first observed through oscillations of the magnetic field at the edge, the so-called Mirnov oscillations.

Due to the singular nature of the perturbation, it is helpful to separate the calculation in thin layers centered on the resonant surfaces from that outside these layers. Asymptotic matching of the solutions in the layers to the exterior solutions completes the determination of the perturbed equilibrium. Within the layer, the MHD model is generally inadequate but the analysis is facilitated by an expansion based on the thinness of the 


\section{$\mathrm{OV} / 2-5$}

layer, $q-m / n \ll 1$. Outside the layer, by contrast, the MHD model gives a good description of the distortion of the equilibrium, but in order to address the effect of the island on the profiles it must be supplemented by a transport calculation. [9]

The helical equilibrium outside the layer is sensitive to the imposed geometry as well as to the current and pressure profiles, so that its calculation generally requires a numerical approach. In nominally symmetric devices, it is appropriate to linearize about the unperturbed equilibrium. The standard code for calculating the perturbed equilibrium is the venerable PEST-III, which calculates both the tearing-parity and twisting-parity (interchange) wave-functions in tokamaks with an axisymmetric boundary condition.[18] A simplified picture of PEST-III's function is that for a given toroidal mode number $n$ and an equilibrium with $M$ singular surfaces, it calculates a matrix relating the $M$ amplitudes $\hat{J}_{m, n}$ of the delta functions in Eq. 1 to the amplitudes of the reconnected flux in the $M$ magnetic islands at the singular surfaces. In the special case where the toroidal mode number $n$ and the profile of the safety-factor are such that the plasma contains only one resonance, the matrix reduces to a single element, the well-known $\Delta^{\prime}$ parameter. [19] An important property of this parameter is that it grows without bounds as the plasma approaches ideal marginal stability (as a result of rising $\beta$, for example). [16] This is a manifestation of the approach of an equilibrium bifurcation. An ongoing effort to replace PEST-III by a code that accounts for the geometry of the coils and uses more advanced numerical techniques is making steady progress. The current embodiment of this effort, the Ideal Perturbed Equilibrium Code (IPEC), calculates the ideal wavefunctions in the presence of resonant magnetic perturbations driven by field errors and control coils. [20] Among other applications, IPEC enables the optimization of the design of error-field correction coils. [21]

In stellarators, by contrast, the reference-state itself contains current singularities but their amplitude is minimized by design. Stellarator equilibrium codes separate into those that assume simply nested magnetic surfaces, such as VMEC, [22] and those that allow for magnetic islands, such as HINT and PIES. [23] Simulations with the HINT code have shown that the geometry of the exterior region can lead to island healing. [24] Such a healing mechanism is distinct from that predicted by the layer theory, the latter depending only on the local value of the magnetic well at the resonant surface. [25, 26, 27] Generally speaking, global island codes serve to identify the qualitative effects of the overall geometry. Their partial or complete omission of effects that are important in the layer, however, such as convective transport near the separatrices, polarization currents, and the role of viscosity on the phase of the island, prevents them from predicting the observed island widths and can lead to differences in their results. [23] Unfortunately, there is no existing framework for coupling global and layer calculations in Stellarators.

Turning our attention to the singular layer, we note that the analysis in this region is facilitated by the fact that, for thin islands, the flute ordering applies:

$$
k_{\|} \ll k_{\perp} .
$$

Note in the nonlinear regime, the flute ordering implies that the non-resonant sidebands are smaller than the resonant harmonics by a factor of $k_{\|} / k_{\perp}$. The flute ordering also 


\section{$\mathrm{OV} / 2-5$}

underlies the gyrokinetic equation, so Eq. 2 implies that gyrokinetic codes are applicable in the layer region. For long wavelength modes $\left(k_{\theta} r_{s} \sim 1\right.$, where $r_{s}$ is the radius of the singular surface), the gradients of the perturbations are approximately perpendicular to the flux surfaces. In principle, this allows for a $1.5 \mathrm{D}$ treatment $[28,29]$ but in practice numerical simulations are often carried out in 2D slab geometry. [30, 31, 32]

The theory divides into two regimes depending on the width of the island. The first regime applies when the width of the island is such that the perpendicular transport dominates over the parallel transport. In this regime, quasi-linear theory applies and leads to the description of the shielding of small resonant magnetic perturbations by flowing plasmas and of the failure of shielding manifested by mode-penetration. The second regime, when parallel transport dominates, describes fully developed islands such as snakes [33] and the islands created in the edge of stellarators. In this second regime, the island modifies the equilibrium profiles giving rise to flattening and sometimes local peaking of the profiles around the O-point. We describe both regimes in turn.

\section{Shielding and penetration of resonant magnetic perturbations}

When the plasma rotation exceeds the rate of reconnection at the resonant surface, reconnection is inhibited. $[34,35,36]$ The resulting perturbation is reduced from its amplitude in the absence of rotation by a factor of $H^{-1 / 3}\left(\omega_{A} / \omega\right)$ where $\omega$ and $\omega_{A}$ are the rotation and Alfvén frequencies, and $H=(\eta \nu)^{-1 / 2}$ is the Hartmann number. The flowing plasma exerts a viscous force on the suppressed island resulting in a phase shift between it and the current. The viscous force is opposed by an electromagnetic force that is concentrated in the resonant layer and acts as a brake on the rotation. This resonant electromagnetic force is augmented by a neoclassical drag that is distributed across the entire plasma. Analysis of braking observations on JET and NSTX indicate that the neoclassical drag dominates the braking process. $[9,37]$

A crucial feature of the resonant braking force is that it exhibits a minimum as a function of the rotation velocity. The viscous force exerted by the plasma, by contrast, is a monotone decreasing function of the rotation velocity that vanishes when the plasma is rotating at its natural velocity, i.e. at its velocity in the absence of resonant magnetic perturbation (RMP). It follows from the shape of the two force curves that the balance between the viscous and electromagnetic forces exhibits a tangent bifurcation as the amplitude of the RMP increases. Beyond the tangent bifurcation point, the island grows rapidly up to a width comparable to the vacuum width. At the same time, the plasma rotation adjusts so as to satisfy the no-slip as well as the frozen-in conditions. The bifurcation is known as mode penetration. [35, 38]

Mode penetration imposes conflicting requirements on the design of the error-field control coils for ITER. Avoidance of low-density locked modes (LDLM) requires minimizing the amplitude of the magnetic perturbations that are resonant in the core. The tolerances for error fields become more stringent during operation above the no-wall stability limit due to the amplification of the braking force in this regime.[39, 40,41] On the other hand, 


\section{$\mathrm{OV} / 2-5$}

a recently developed technique for the mitigation of Edge Localized Modes (ELM) relies on the application of magnetic perturbations tailored so as to maximize the amplitude of the edge-resonant harmonics. such perturbations act to degrade the H-mode pedestal and thereby diminish or completely eliminate ELMs. [42, 43] The importance of ELM mitigation for ITER is motivating intense interest in the modeling of mode penetration and rotation braking by RMPs. An intriguing feature of the observations is the preservation and sometimes steepening of the electron temperature pedestal during the application of the RMP. A possible explanation of this effect is that the very high electron diamagnetic flows in the electron pedestal screens the perturbation, locally preserving good flux surfaces. [44]

At the onset of mode-penetration, the effect of the RMP on the profiles other than that of velocity is small. As the island grows to saturation, however, it modifies the remaining profiles. The next section describes the interaction between the profiles and the magnetic island in the case of penetrated RMP as well as for spontaneous tearing modes such as the NTM.

\section{Transport in magnetic islands}

The primary question regarding relaxed magnetic islands is their effect on the profiles of density, temperature, current, and rotation velocity. The changes in the profiles determine the effect of the island on overall confinement, and they determine whether the island grows or decays. Our understanding of the effect of the profiles on the island amplitude informs the methods used for avoiding islands as well as for suppressing them with RF current-drive and heating.

To evaluate helical equilibria with islands, the first obstacle we must confront is the interdependence of the profiles and the island width. Fortunately, in ideally stable equilibria the perturbed helical current is small compared to the background current. As a result, the variation across the layer of the resonant Fourier component of the helical flux, $\tilde{\psi}_{m, n}$, is small. The approximation that consists in neglecting this variation is known as the "constant- $\tilde{\psi}$ " approximation. This approximation greatly facilitates the analysis and interpretation of observations. It implies that just two parameters specify the magnetic geometry: the amplitude $\tilde{\psi}_{m, n}$ and phase $\phi$ of the resonant perturbation (for a rotating island, $d \phi / d t=\omega t)$. The helical flux takes the form

$$
\psi=B_{0} x^{2} / 2 L_{s}+\tilde{\psi}_{m, n} \cos (m \theta-n \zeta-\phi),
$$

where $x$ is the distance from the resonant surface, $L_{s}$ is the shear length, and $B_{0}$ is the

background field. Equation (3) implies that the width of the island is $W=4 \sqrt{\tilde{\psi}_{m, n} L_{s} / B_{0}}$.

The constant- $\tilde{\psi}$ approximation leads to the following simple expression of the matching condition between the resonant layer and the exterior solution:

$$
\Delta_{m, n}^{\prime} \tilde{\psi}_{m, n}=\int d x \oint \frac{d \theta}{\pi} J \exp [i(m \theta-n \zeta-\phi)]
$$




\section{$\mathrm{OV} / 2-5$}

where $\Delta_{m, n}^{\prime}$ is the matching parameter from the exterior solution. The helical current in Eq. (4) includes the perturbed conduction, bootstrap, polarization, Pfirsch-Schlüter, and the beam- and RF-driven currents. A solution method is now apparent: the first step consists in solving the transport problem in the geometry specified by Eq. (3). The second step consists in using the result of the transport calculation to evaluate the integral in Eq. (4). Separating the contributions to the current from the time derivatives of $\tilde{\psi}_{m, n}$ and $\phi$ leads to the evolution equations for the components respectively in phase and in phase-quadrature with the reconnected flux,

$$
\begin{aligned}
\frac{\tau_{R}}{r_{s}^{2}} \frac{d W}{d t} & =\Delta^{\prime}+\Delta(W, \omega) \\
\frac{d^{2} \phi}{d t^{2}} & =F_{t}(W, \omega)
\end{aligned}
$$

The above two equations generalize the results of Rutherford. [45] The two functions $\Delta(W, \omega)$ and $F_{t}$ represent respectively the free energy available in the layer for reconnection and the acceleration of the island caused by any imbalance of lateral forces in the layer. The determination of these two functions is the central task of the singular-layer theory. In the remainder of this section we describe some of the recent progress in our knowledge of the contributions of the pressure-driven (Pfirsch-Schlüter and bootstrap) currents and of the inertia-driven (polarization) currents to these two functions.

The contributions of the Pfirsch-Schlüter [46, 25] and Neoclassical current $[47,48]$ to the island evolution have long been well understood in the limit of large islands. The increasing resolution in the observations of electron temperature profiles, however, have stimulated theoretical research on the degree of temperature flattening. The effects of a magnetic island on the profiles of temperature are governed by the competition between parallel transport and perpendicular transport. The role of this competition in magnetic islands is analogous to that in the scrape-off layer. The controlling parameter is the connection length with respect to the magnetic axis of the island. This is the length taken to circumnavigate a helical flux surface along a field line. Near the separatrix, the connection length becomes very large, reflecting the fact that the magnetic field near the X-lines is nearly parallel to the magnetic axis of the flux tube. For sufficiently long connection lengths, the parallel fluxes are no longer effective in relaxing the profiles along the field line. Equilibrium gradients may then develop within the flux surfaces.

Near the O-point of the island, the connection length $L_{c} \sim L_{s} / k W$ where $L_{s}$ is the magnetic shear length. It follows that as the island grows, there is a characteristic width above which the parallel transport dominates and the gradients become perpendicular to the flux surfaces. In the collisional regime, the equation describing the transport near the island is [45]

$$
\kappa_{\|} \nabla_{\|}^{2} T+\kappa_{\perp} \nabla_{\perp}^{2} T=0
$$

The resulting critical width is [49]

$$
W_{c} \sim\left(\frac{\kappa_{\|}}{\kappa_{\perp}} \frac{L_{s}}{k_{\theta}}\right)^{1 / 4} .
$$




\section{$\mathrm{OV} / 2-5$}

In the collisionless regime, by contrast, the drift-kinetic equation governs the transport,

$$
v_{\|} \nabla_{\|} f+\nabla_{\perp}\left(D \nabla_{\perp} f\right)=0 .
$$

Here $D$ is a phenomenological quasilinear diffusion coefficient describing the effect of turbulent transport or of the mode itself. [50] The critical width is then [51]

$$
W_{c}=\left(\frac{D L_{s}}{k_{\theta} v_{t}}\right)^{1 / 3} .
$$

For $W \gg W_{c}$, the profiles inside the separatrix are flat in the absence of sources or inward pinches. Profile flattening is indeed routinely observed in experiments, $[52,53]$ but there are also challenging observations of profile peaking inside large islands. [54] Except for the observations of snakes in the core where the constant- $\tilde{\psi}$ approximation fails, [33] most of the observations of peaking are for islands near the edge where the effects of sources such as radiation and charge exchange are likely to play a role. The cause of profile peaking is presumably a combination of very good confinement and an inward pinch, but there is scant existing theory.

Another contribution to the island evolution integrals (5)-(6) that has received recent attention is that of the polarization current. The corresponding theory applies to rotating as well as locked islands, and to islands located in the core as well as in the edge. The polarization current results from the acceleration of the plasma as it flows along the Laval nozzle formed by the distorted flux surfaces outside the sepraratrix. The vector product of the acceleration and the magnetic field gives rise to a well-known ion drift with non-vanishing divergence. A parallel current must accompany this drift to maintain quasi-neutrality. This parallel current mediates the effect of the plasma flows on the island evolution.

In order to determine the distribution of the polarization current, it is necessary to know the velocity of the plasma flow with respect to the island. Since the ions and electrons experience opposite diamagnetic drifts, they cannot both be at rest with respect to the island. In order to facilitate the discussion of the relative velocity of the island with respect to the electron and ion fluids, it is convenient to express all velocities in a frame where the background electric field vanishes. In this frame it is straightforward to show that the velocity of the island depends on the degree of profile flattening inside the separatrix. [55] This follows from the frozen-in and the no-slip conditions. The frozen-in condition expresses the fact that the separatrix traps the electron fluid whenever $W>\rho_{s} \sqrt{C}$ where $C=\left(L_{s} / L_{n}\right)^{2}\left(\nu_{e} / \omega_{* e}\right)\left(m_{e} / m_{i}\right)<1$ is a measure of the collisionality (the bound $C<1$ corresponds to the semi-collisional regime that describes most fusion experiments). [56] The no-slip condition, by contrast, expresses the fact that the much greater viscosity of the ion fluid compared to the electron fluid results in the continuity of the ion velocity across the separatrix. These two conditions lead to the following expression, illustrated in Fig. 1 for the island velocity $u$ :

$$
u=v_{D e}^{\text {in }}+v_{D i}^{\text {out }}-v_{D i}^{\text {in }}
$$

where the $v_{D s}$ are the diamagnetic drifts, the index $s=i, e$ labels the species, and the superscripts indicate on which side of the separatrix the quantity is measured. To 
avoid unnecessarily burdening the notation, we assume that the island is sufficiently wide that the electron temperature is fully flattened inside the separatrix. We further assume that the degree of flattening of the ion temperature is equal to that of the density. The diamagnetic velocities are then

$$
v_{D s}^{\text {in }}=f v_{D s}^{\text {out }}
$$

where $f$ is a flattening factor varying between 0 and 1 that describes the ratio of the density gradient at the O-point of the island to the density gradient in the reference state. Substituting the diamagnetic drifts into the equation for the island velocity yields

$$
u=f v_{* e}^{\text {out }}+(1-f) v_{* i}^{\text {out }} \text {, }
$$

We see that islands that are sufficiently thin for the density and ion temperature gradient to be maintained $(f=1)$ propagate at the electron diamagnetic drift velocity. Note that electrons outside the separatrix, however, are propagating at $1+\eta_{e}$ times the diamagnetic velocity, where $\eta_{s}$ is the ratio of the background density scale-length and the temperature scale-length of species $s$. In the opposite limit of large islands in which the ion pressure is fully flattened $(f=0)$, the island propagates at the ion pressure drift velocity, or $-\left(1+\eta_{i}\right) \tau \omega_{* e}$ where $\tau=T_{i} / T_{e}$ is the temperature ratio. That is, the ions outside the separatrix are at rest with respect to the island.

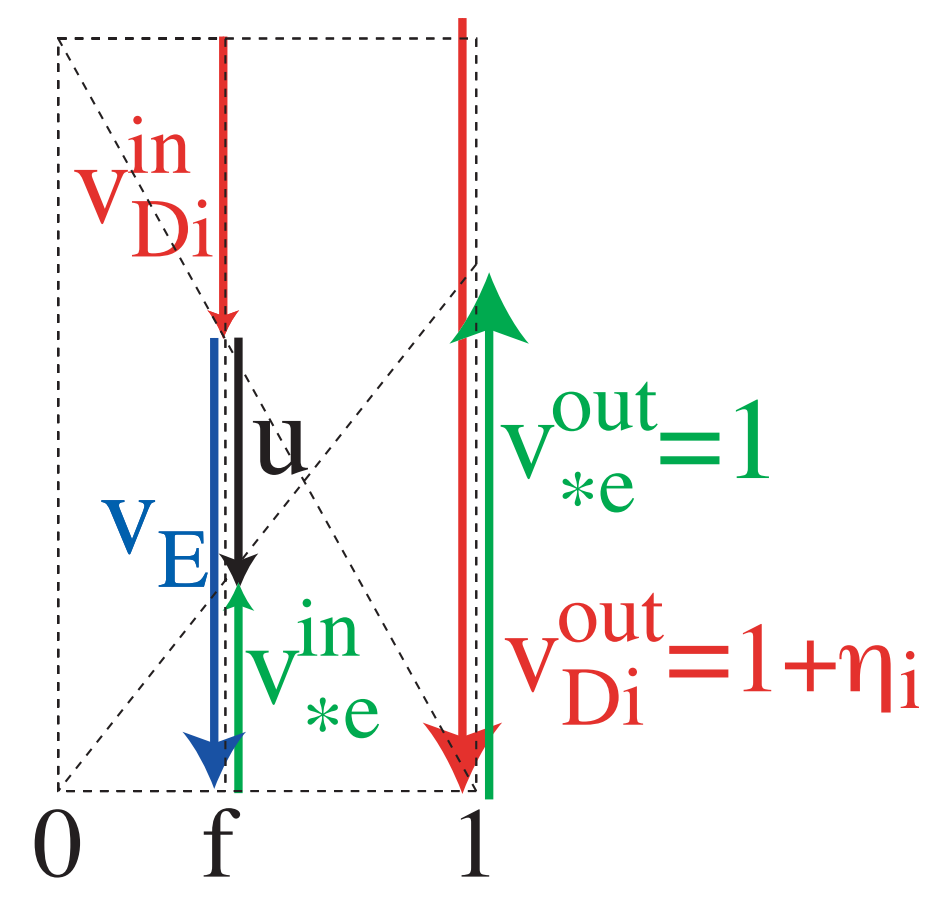

Fig. 1: Graphical solution for the island propagation velocity in terms of the profile-flattening factor $f$.

The above discussion leaves unanswered the question of the characteristic width $W_{f}$ for density flattening. This question is controversial and estimates have differed by more than an order of magnitude, from the semi-collisional boundary $W_{f} \sim \rho_{s} \sqrt{C}$ [31] to the drift-acoustic width $W_{f} \sim \rho_{s} L_{s} / L_{n}$ at which the drift wave couples with ion-acoustic waves.[57] Numerical results for $C<1$, however, suggest that the coupling of the island to 


\section{$\mathrm{OV} / 2-5$}

drift waves can flatten the profiles even in the absence of ion-acoustic waves, for $k_{\|} c_{s} \ll \omega_{*}$. An estimate consistent with the available theoretical results is $W_{f} \sim \rho_{s} \max \left(1, C^{1 / 2}\right)$. This estimate is qualitatively consistent with observations in LHD showing that the electric field inside the $m=n=1$ island drops to zero for $W \approx 1 \mathrm{~cm}$. $[58,59]$ It is also qualitatively consistent with other observations in the same experiment showing a clear increase of the iso-density width with collision frequency and a decrease with $\beta$. [53] Lastly, measurements of the rotation velocity of neoclassical tearing modes in DIII-D have found that the islands were rotating in the ion direction, consistent with ion pressure flattening. $[60]$

It is natural to expect the polarization drift to become vanishingly small for island of width $W \gtrsim W_{f}$, since these islands are co-rotating with the ions. Surprisingly, numerical simulations in the collisional regime, $C \gg 1$, show that this is not the case. [32] Instead, the polarization current exhibits a pronounced resonance when the island width is such that the drift wave couples strongly to ion-acoustic waves. That is, the resonance occurs for $\omega_{*}=k_{\|} c_{s}$, or $W=\rho_{s} L_{s} / L_{n}$. Analysis of the diffusion of momentum in an island with fully flattened profile shows that the resonance manifests itself as a singularity of the Reynolds stress appearing in the diffusion equation. [61] This is reminiscent of the role of sonic flows in the scrape-off layer. In the case of islands, the resonance is robustly stabilizing and may determine the effective threshold for the onset of neoclassical tearing modes.

Recent investigations have examined the interaction between turbulence and magnetic islands. McDevitt and Diamond have calculated the evolution of the drift-wave population density in the presence of a tearing mode using the wave-kinetic equation to model the turbulence. They concluded that the turbulence gives rise to a negative viscosity that acts as a pump on the resonant long-wavelength mode [62]. The negative viscosity results in asymptotic oscillations of the wave-function caused by the coupling of the tearing mode to zonal flows. Ishizawa et al. have carried out numerical simulations of the evolution of the double tearing mode in the presence of electromagnetic turbulence. They found that in a first stage, the zonal flows driven by the turbulence stabilize the linearly unstable double-tearing mode. In a second stage, however, long wavelength magnetic fluctuations exert a drag that suppresses the zonal flows, leading to the rapid growth of the double tearing mode [63]. The effect of noise on NTM seeding has also been investigated by Itoh et al. [64]. Lastly, Militello et al. have shown that turbulence reduces the propagation velocity of the island. [65] Its effect is destabilizing for thin islands but becomes stabilizing, primarily due to the reduced propagation velocity, for islands greater than a few times the Larmor radius.

\section{Discussion}

The control of magnetic islands has progressed to the extent that they no longer present a substantial threat to the confinement of fusion plasmas. As a result, the focus of research has shifted to using islands as the agents of plasma control systems. Examples include the use of islands for driving current in the core, [8] for mitigating ELMs, [42] and for controlling transport in the edge of stellarators. [52] 
Theory has played an important role in developing our present abilities with regard to magnetic islands. In addition to predicting pressure-driven healing in stellarators [25] and the growth of neoclassical tearing modes in tokamaks, [47] theoretical understanding has guided the development of techniques for using RF current drive to suppress islands. [66] Although analytic results and simulations have proven to be useful guides for interpreting experiments and providing qualitative predictions, much work remains to be done in order to achieve quantitative prediction capabilities. The renewed interest in the development of computational tools for predicting the effects of external coils on three-dimensional waveforms [20] is encouraging in view of the limitations affecting initial value codes. In the near term, however, the progress demonstrated by initial value codes with regard to the performance of two-fluid time steppers [14, 15] and anisotropic heat transport [67, 68] is likely to place them at the forefront of advances in our understanding of island physics.

Research funded by the US Department of Energy under contracts \# DE-FG03-96ER-54346 and DE-FC02-04ER54785.

\section{References}

[1] ZOHM, H., Phys. Plasmas 4 (1997) 3433.

[2] GAntenbein, G. et al., Phys. Rev. Lett. 85 (2000) 1242.

[3] PETTY, C. C. et al., Nucl. Fusion 44 (2004) 243.

[4] ISAYAMA, A. et al., Nucl. Fusion 47 (2007) 773.

[5] YU, Q. et al., Phys. Plasmas 11 (2004) 1960.

[6] HUMPhreYS, D. A. et al., Phys. Plasmas 13 (2006) 056113.

[7] WESTERHOF, E. et al., Nucl. Fusion 47 (2007) 85.

[8] CHU, M. S. et al., Phys. Plasmas 13 (2006) 114501.

[9] LAZZARO, E. et al., Phys. Plasmas 9 (2002) 3906.

[10] LA HAYE, R. J. et al., Phys. Plasmas 1 (1994) 373.

[11] LA HAYE, R. J. et al., Nucl. Fusion 33 (1993) 349.

[12] LA HAYE, R. J. et al., Phys. Fluids B 4 (1992) 2098.

[13] SUGIYAMA, L. E. et al., Phys. Plasmas 7 (2000) 4644.

[14] STRAUSS, H. et al., Nucl. Fusion 44 (2004) 1008.

[15] MURPHY, N. A. et al., Phys. Plasmas 15 (2008) 042313.

[16] BREnNAN, D. P. et al., Phys. Plasmas 9 (2002) 2998. 
[17] BRENNAN, D. P. et al., Phys. Plasmas 10 (2003) 1643.

[18] GRIMM, R. C. et al., Resisitve instabilities in tokamak geometry, in Plasma Physics and Controlled Nuclear Fusion Research, 1982, volume 3, pages 35-47, International Atomic Energy Agency, Vienna, 1983.

[19] FURTH, H. P. et al., Phys. Fluids 16 (1973) 1054.

[20] PARK, J.-K. et al., Phys. Plasmas 14 (2007) 052110.

[21] PARK, J.-K. et al., Nucl. Fusion 48 (2008) 045006 (8pp).

[22] HIRShMAN, S. P. et al., J. Comp. Phys. 96 (1991) 99.

[23] NAKAMURA, Y. et al., Nucl. Fusion 44 (2004) 387.

[24] NARIHARA, K. et al., Phys. Rev. Lett. 87 (2001) 135002.

[25] CARY, J. R. et al., Phys. Fluids 28 (1985) 1392.

[26] HEGNA, C. C. et al., Phys. Fluids B: Plasma Physics 1 (1989) 392.

[27] BHATtACHARJEE, A. et al., Phys. Plasmas 2 (1995) 883.

[28] CONNOR, J. W. et al., Phys. Plasmas 8 (2001) 2835.

[29] WAELBROECK, F. L. et al., Phys. Rev. Lett. 87 (2001) 215003.

[30] BISKAMP, D., Nucl. Fusion 19 (1979) 777.

[31] OTtaViani, M. et al., Phys. Rev. Lett. 93 (2004) 075001.

[32] FITZPATRICK, R. et al., Phys. Plasmas 13 (2006) 122507.

[33] WESsOn, J. A., Plasma Phys. and Controlled Fusion 37 (1995) A337.

[34] FITZPATRICK, R. et al., Phys. Fluids B 3 (1991) 644.

[35] FITZPATRICK, R., Nucl. Fusion 33 (1993) 1049.

[36] BOOZER, A. H., Phys. Plasmas 3 (1996) 4620.

[37] ZHU, W. et al., Phys. Rev. Lett. 96 (2006) 225002.

[38] FISHPOOL, G. M. et al., Nucl. Fusion 34 (1994) 109.

[39] LA HAYE, R. J. et al., Nucl. Fusion 32 (1992) 2119.

[40] GarofalO, A. M. et al., Phys. Rev. Lett. 82 (1999) 3811.

[41] GAROFAlO, A. M. et al., Nucl. Fusion 42 (2002) 1335.

[42] EVANS, T. E. et al., Physical Review Letters 92 (2003) 235003.

[43] FEnStermaCHER, M. E. et al., Phys. Plasmas 15 (2008) 056122. 
[44] NARDON, E. et al., Private communication (2008).

[45] RUTherforD, P. H., Phys. Fluids 16 (1973) 1903.

[46] KOTSCHEnREuther, M. et al., Phys. Fluids 28 (1985) 294.

[47] CARRERA, R. et al., Phys. Fluids 29 (1986) 899.

[48] HEGNA, C. C., Phys. Plasmas 5 (1998) 1767.

[49] FITZPATRICK, R., Phys. Plasmas 2 (1995) 825.

[50] HAZELTine, R. D. et al., Phys. Rev. Lett. 37 (1976) 102.

[51] HAZELtine, R. D. et al., Phys. Plasmas 4 (1997) 2920.

[52] INAGAKI, S. et al., Phys. Rev. Lett. 92 (2004) 055002.

[53] TANAKA, K. et al., Plasma Phys. Control. Fusion 44 (2002) A231.

[54] DE VRIES, P. C. et al., Nucl. Fusion 37 (1997) 1641.

[55] WAELBROECK, F. L., Phys. Rev. Lett. 95 (2005) 035002.

[56] DRAKE, J. F. et al., Phys. Fluids 26 (1983) 2509.

[57] SCOTT, B. D. et al., Phys. Fluids 28 (1985) 275.

[58] IDA, K. et al., Phys. Rev. Lett. 88 (2002) 015002.

[59] IDA, K. et al., Plasma Phys. and Control. Fusion 44 (2004) 290.

[60] LA HAYE, R. J. et al., Phys. Plasmas 10 (2003) 3644.

[61] FITZPATRICK, R. et al., Phys. Plasmas 12 (2005) 082510.

[62] MCDEVITT, C. J. et al., Phys. Plasmas 13 (2006) 032302.

[63] ISHIZAWA, A. et al., Phys. Plasmas 14 (2007) 040702.

[64] ITOH, S.-I. et al., Plasma Phys. Control. Fusion 46 (2004) 123.

[65] MILtellO, F. et al., Phys. Plasmas 15 (2008) 050701.

[66] HEGNA, C. C. et al., Phys. Plasmas 4 (1997) 2940.

[67] SOVINEC, C. R. et al., J. Comp. Phys. 195 (2004) 355.

[68] GUEnTER, S. et al., J. Comp. Phys. 209 (2005) 354. 